\title{
The value of multidisciplinary team (MDT) management in the diagnosis and treatment of primary intrathoracic synovial sarcomas: a single-center experience
}

\author{
Huayu He ${ }^{1 \#}$, Lin Yang ${ }^{2 \#}$, Yue Peng ${ }^{1}$, Li Liu $^{2}$, Lei Liu ${ }^{1}$, Qi Xue ${ }^{1}$, Shugeng Gao ${ }^{1}$ \\ ${ }^{1}$ Department of Thoracic Surgery, National Cancer Center/National Clinical Research Center for Cancer/Cancer Hospital, Chinese Academy \\ of Medical Sciences and Peking Union Medical College, Beijing, China; ${ }^{2}$ Department of Pathology, National Cancer Center/National Clinical \\ Research Center for Cancer/Cancer Hospital, Chinese Academy of Medical Sciences and Peking Union Medical College, Beijing, China \\ Contributions: (I) Conception and design: H He, L Yang; (II) Administrative support: S Gao; (III) Provision of study materials or patients: H He, \\ L Yang, Y Peng; (IV) Collection and assembly of data: H He, L Liu, L Liu; (V) Data analysis and interpretation: H He, L Yang, Q Xue; (VI) \\ Manuscript writing: All authors; (VII) Final approval of manuscript: All authors. \\ \#These authors contributed equally to this work. \\ Correspondence to: Shugeng Gao. Department of Thoracic Surgery, National Cancer Center/National Clinical Research Center for Cancer/Cancer \\ Hospital, Chinese Academy of Medical Sciences and Peking Union Medical College, Beijing, China. Email: gaoshugeng@vip.sina.com.
}

Background: Synovial sarcoma (SS) is a rare malignant soft tissue tumor. Primary intrathoracic SS is extremely rare, with limited diagnosis and treatment experiences. The aim of our study was to retrospectively study the clinicopathological characteristics, treatment and prognosis of primary intrathoracic SS and the impact of multidisciplinary team (MDT) management in diagnosis and treatment on patient prognosis.

Methods: The clinical and pathological characteristics, treatment, survival and prognosis of patients with primary intrathoracic SS admitted to the National Cancer Center from January 1999 to December 2018, as well as MDT intervention during diagnosis and treatment, were retrospectively analyzed.

Results: Thirteen patients were enrolled, including 7 (53.8\%) males and 6 (46.3\%) females, with primary intrathoracic SS in the lung $(8 / 13,61.5 \%)$, mediastinum $(4 / 13,30.8 \%)$ and pleura $(1 / 13,7.7 \%)$ as confirmed by morphological observation, immunohistochemical (IHC) staining and fluorescence in situ hybridization (FISH). Overall, 10/13 (76.9\%) patients underwent surgery, and 6/10 (60.0\%) received postoperative adjuvant therapy. Only $23.1 \%$ of patients received nonsurgical therapy. The MDT discussed and managed seven patients before and/or after surgery and one patient who did not undergo surgery. The estimated 3and 5 -year overall survival (OS) rates were $50.0 \%$ and $30.0 \%$, respectively. Patients who were managed by an MDT had a longer median OS time than those who were not (46.0 vs. 18.0 months). Age ( $\mathrm{P}=0.018)$, tumor location $(\mathrm{P}=0.029)$, and $\mathrm{Ki}-67(\mathrm{P}=0.020)$ were found to be significantly related to OS.

Conclusions: Monophasic morphology and fusion gene characteristics are the main features for the diagnosis of primary intrathoracic SS. MDT management can help obtain accurate diagnoses and provide reasonable therapeutic options.

Keywords: Synovial sarcoma (SS); pleura; lung; mediastinum; multidisciplinary team (MDT)

Submitted Sep 14, 2020. Accepted for publication Nov 20, 2020.

doi: $10.21037 /$ jtd-20-2887

View this article at: http://dx.doi.org/10.21037/jtd-20-2887

(c) Journal of Thoracic Disease. All rights reserved. 


\section{Introduction}

Soft tissue sarcomas (STSs) are a rare group of mesenchymal tumors, accounting for only approximately $1 \%$ of all adult malignant tumors (1). Synovial sarcoma (SS) accounts for approximately $2.5-10 \%$ of STSs (2). SS is mainly reported to occur in the soft tissues near the joints of the lower and upper limbs, especially the knee joint $(3,4)$ and in the head and neck (5), but rarely in the intrathoracic cavity. SS accounts for $14.7 \%$ of all primary intrathoracic sarcomas in the lung, pleura and mediastinum and is also the most common type of primary intrathoracic sarcoma (6). Most cases of primary intrathoracic SS occur in the pleura and lung, and those in the heart and mediastinum are rare (6-10). Because of the rarity of primary intrathoracic SS, there are obvious challenges and a lack of experience in its clinical diagnosis and treatment. For instance, pathologically, resectable SS in soft tissue is not difficult to diagnose by assessing enough specimens under the microscope, but the differential diagnosis is still difficult in those with atypical mediastinal morphology, especially for those with small round cell and spindle cell tumors, sarcomatoid mesothelioma, fibrosarcoma, leiomyosarcoma, or other tumors, as well as for small specimens that can only be obtained by puncture and not surgery $(11,12)$.

The challenge of diagnosing and treating primary intrathoracic SS (13) is mainly the result of the location of the tumor and the difficulty of the operation, which requires a safe margin around the soft tissue tumor (generally ensuring that the normal tissue at least $2 \mathrm{~cm}$ away from the tumor edge is removed). Therefore, multidisciplinary partnerships are particularly important for discussing diagnosis and treatment plans. However, to the best of our knowledge, the literature on primary intrathoracic SS mainly comprises sporadic case reports, with few relevant conclusions about diagnosis and treatment. The purpose of this study is to summarize and discuss the diagnosis and treatment of primary intrathoracic SS from the perspective of multidisciplinary team (MDT) collaboration by analyzing 13 cases of primary intrathoracic SSs. We present the following article in accordance with the STROBE reporting checklist (available at http://dx.doi.org/10.21037/jtd-202887).

\section{Methods}

\section{Patients}

We retrospectively reviewed a database for the clinical records of 13 consecutive patients with primary intrathoracic SS between 1999 and 2018 at National Cancer Center/Cancer Hospital, Chinese Academy of Medical Sciences, and Peking Union Medical College. The inclusion criteria of our study were as follows: (I) histologically confirmed primary intrathoracic SS, including tumors involving the lung, pleura, mediastinum and heart; and (II) detailed clinical data. The exclusion criteria were as follows: (I) histological diagnoses considered suspicious or unconfirmed by the pathology department of our institute; (II) metastatic tumors from other sites; and (III) a lack of detailed clinical information and follow-up information. In total, 56 patients were excluded from this study. The entire enrollment process is clearly shown in Figure 1. Among these patients, 38 patients had secondary SSs; 13 patients were not confirmed by fluorescence in situ hybridization (FISH); 3 patients had incomplete medical data; and 2 patients were lost to follow-up. Finally, a total of 13 patients were enrolled in the present study (Figure 1).

Our study protocol for this retrospective study was approved by the ethics committees of our center (Approval number: 20/210-2406) and was performed strictly in accordance with the Declaration of Helsinki (as revised in 2013). The requirement for informed consent was waived because of the retrospective nature of this study. Patients were followed up in the outpatient department every 3-6 months for the first two years after surgery and then annually thereafter. The follow-up included medical history assessments, physical examinations, and chest computed tomography (CT). The last follow-up ended on August 31, 2019. The primary endpoint of our study was the 5 -year overall survival (OS) rate.

\section{Clinicopathological parameters}

The clinicopathological parameters of the patients, including age, sex, smoking history, tumor histopathology, tumor size, CT imaging features, lymph node metastasis, distant metastasis, and TNM stage, were obtained from the medical records. The pathological classification of the primary tumor, degree of lymph node metastasis, and presence of distant metastasis were assessed based on the 8th edition TNM staging system. MDT was defined as collaboration among different professionals involved in the diagnosis and treatment of cancer patients, including surgeons, physicians, radiotherapy specialists, radiologists, pathologists, and nurses. Whether an MDT discussion occurred during the diagnosis and treatment of the patients 


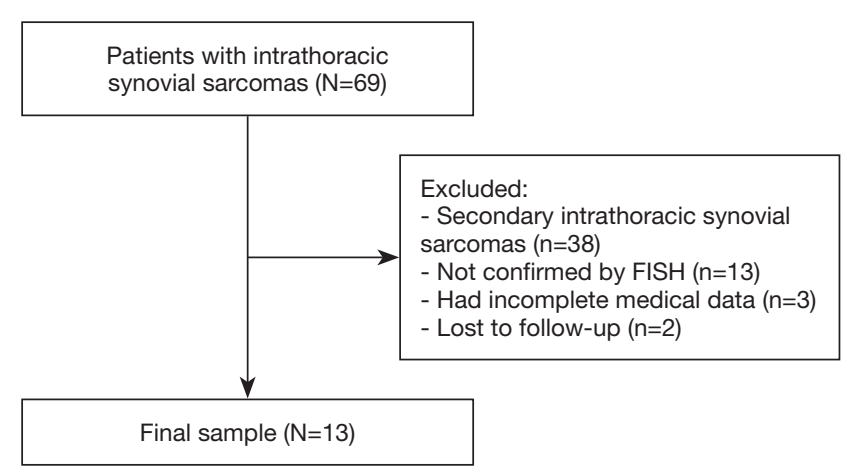

Figure 1 Flowchart of the exclusion criteria and study design.

was mainly determined based on medical records.

For all 13 patients, immunohistochemical (IHC) staining was performed on formalin-fixed and paraffinembedded tissue sections. SS18 on chromosome 18q11.2 was analyzed by FISH. To reduce inaccuracies or errors in pathological diagnosis, the results were scored blindly by two pathologists based on 100 cells for each case.

\section{Statistical analysis}

SPSS version 23.0 (IBM Corp., Armonk, NY, USA) was used to perform all statistical analyses. Categorical variables were compared with the Pearson chi-square test. KaplanMeier plots were used to depict recurrence-free survival (RFS) and OS. The log-rank test was used to assess differences between groups. The Cox proportional hazard model was used for multivariate analysis, and hazard ratios (HRs) with 95\% confidence intervals (CIs) were used to quantify the strength of the association between predictors and survival. $\mathrm{P}<0.05$ was considered statistically significant. Because SS is a rare disease, the number of patients is limited; however, the data included in the survival analysis are relatively complete, and no important data are missing.

\section{Results}

\section{Patient characteristics}

In total, 13 patients underwent treatment for primary intrathoracic SS at our center in the last twenty years. The baseline characteristics of all patients are summarized in Table 1, including age, sex, smoking history, initial symptoms, tumor location, maximum tumor diameter, CT imaging features, TNM stage, and treatment. Of the 13 patients, $8(61.5 \%)$ had primary pulmonary SS, 4 (30.8\%) had primary mediastinal SS, and 1 (7.7\%) had pleural SS.

\section{Clinicopathological features of primary intrathoracic SS}

Ten of the 13 patients underwent curative intent surgery, and the other three patients were diagnosed by biopsy without surgery. As shown in Table 2, the histologic morphology results showed that $9(75.0 \%)$ tumors were monophasic, 2 (16.7\%) tumors were biphasic, and $2(16.7 \%)$ tumors were poorly differentiated. By immunohistochemistry, $\mathrm{CK}$, vimentin, $\mathrm{AE} 1 / \mathrm{AE} 3$ and Bcl2 were positive, which strongly supported the diagnosis of SS. In addition, the negative CD34 and S100 results excluded solitary fibrosarcoma and malignant peripheral neurilemmoma, respectively. All 13 tumors showed the chromosomal translocation $\mathrm{t}(\mathrm{X} ; 18)(\mathrm{p} 11 ; \mathrm{q} 11)$ typical of SS. Based on this strong evidence, these patients were finally diagnosed with primary SS, which is different from other spindle cell tumors.

\section{Treatment and survival analysis}

Of the 13 patients, 10 underwent surgery, and 3 received radiotherapy or chemotherapy after biopsy. Among the patients who received surgical treatment, there were 7 cases of primary pulmonary SS, 2 cases of primary mediastinal SS and 1 case of primary pleural SS (Table 1).

All 13 patients were successfully followed up. The median follow-up time was 28 months, ranging from 13 to 100 months. The median RFS of patients was 13 months (range, 5-100 months). The estimated 2-, 3- and 5 -year OS rates were $58.3 \%, 50.0 \%$ and $30 \%$, respectively (Figure 2). Only 2 patients were stable after the operation, and the remaining patients had tumor recurrence or progression. Of the 13 patients, $8(61.5 \%)$ died, and 5 $(38.5 \%)$ are still alive, with a median RFS of 13 months (range, 5-100 months). The most common types of disease progression were intrathoracic recurrence and metastasis (7/11), followed by bone metastasis (3/11). There were 2 patients with brain metastasis, all of whom died, and one of them had multiple metastases in the chest and lung at the same time. There were 3 patients with primary site recurrence and progression, and they were still alive by the end of follow-up. Four patients with primary mediastinal SS died: two died of heart failure, one died of brain metastasis, and one died of respiratory failure. One case of primary SS of the pleura recurred 8 months after the operation. The tumor was resected again by surgery with extended margins 


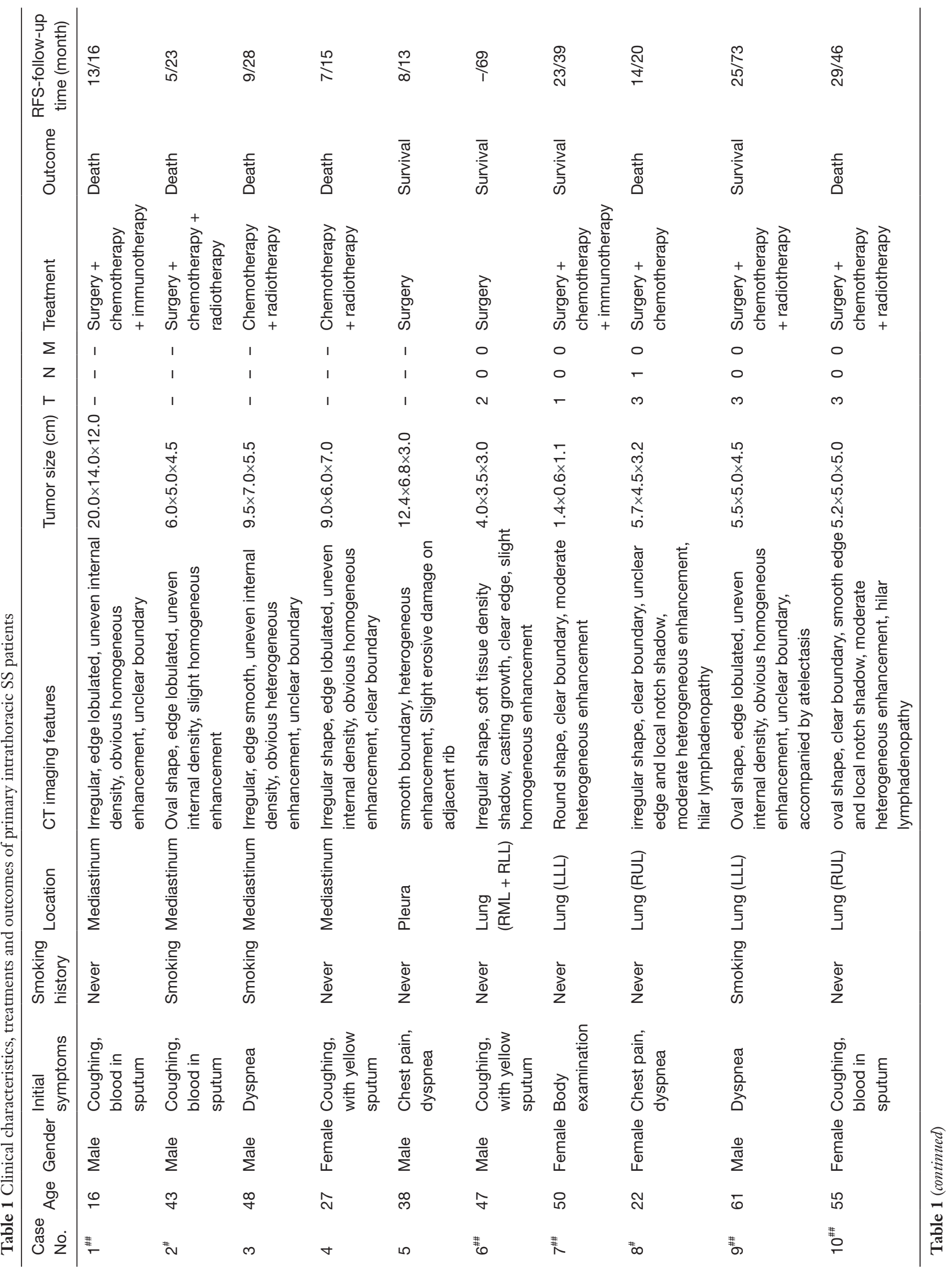



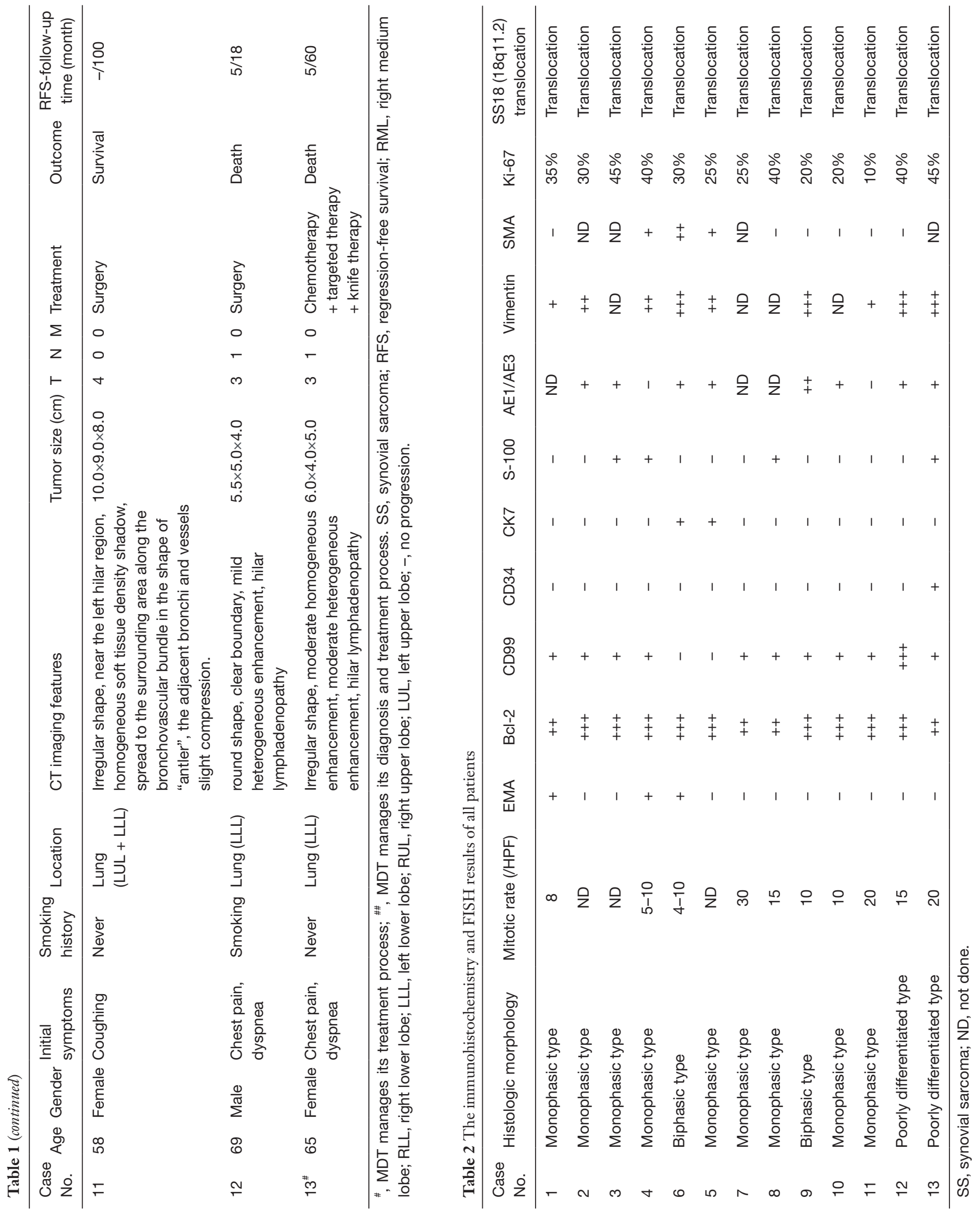


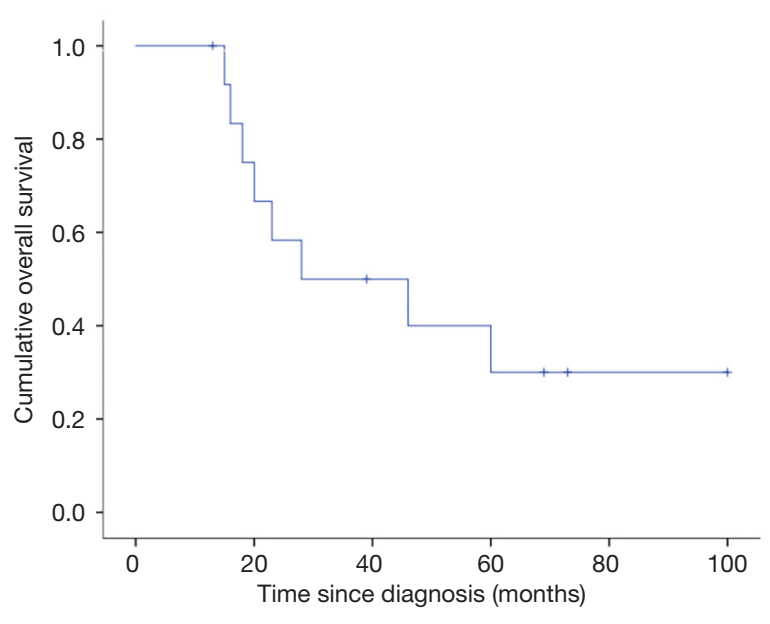

Figure 2 Kaplan-Meier estimate of the OS rate of patients with primary intrathoracic SS after diagnosis. SS, synovial sarcoma.

and adjuvant chemotherapy. Of the 8 patients with primary intrathoracic SS, 4 died of multiple organ failure caused by multiple metastasis of the tumor, 3 died of respiratory failure, and one died of intracranial hypertension caused by brain metastasis.

As shown in Table 3, in univariate Cox regression analysis, three factors were significantly associated with OS: age $(\mathrm{P}=0.018)$, tumor location $(\mathrm{P}=0.029)$, and Ki-67 $(\mathrm{P}=0.020)$. However, there was no statistically significant difference among these factors based on the multivariate analysis.

\section{The value and importance of an MDT}

In our study, 5 patients were discussed and prediagnosed by an MDT during the diagnosis process, and an individualized treatment plan was then formulated. Three patients were discussed in an MDT meeting to design a treatment plan after a definite diagnosis was obtained. Under the guidance of the MDT, 4 patients with primary pulmonary SS received sequential or concurrent radiotherapy and chemotherapy after surgery, and one patient even received immunotherapy after relapsing later. One patient who underwent combined right upper and middle lobectomy did not receive further adjuvant therapy. The diagnosis of one patient was confirmed by biopsy, and this patient received standard chemotherapy followed by molecular targeted therapy. Among the 4 patients with primary mediastinal SS, 2 patients were surgically treated with the aid of the MDT and received concurrent or sequential radiotherapy and chemotherapy postoperatively.
The only patient with a primary pleural SS in our study was a 38-year-old woman who was hospitalized because of chest pain and progressive dyspnea. We found that there was a large oval mass in her left thoracic cavity on chest CT, $12.4 \mathrm{~cm} \times 6.8 \mathrm{~cm} \times 3.0 \mathrm{~cm}$ in size, with smooth boundaries and heterogeneous enhancement after the administration of a contrast agent. Slight erosive damage was found in the adjacent ribs (Figure 3). Solitary fibroma was considered preoperatively and was not discussed at the MDT meeting. Surgical treatment was performed according to the principle of treating solitary fibroma, but the final pathological diagnosis was monophasic-type pleural SS. The immunohistochemistry results showed Bcl-2 (3+), EMA (focal+), CK7 (focal+), Vimentin (3+), SMA (2+), CD34 (-) and S-100 (-). Additionally, the Ki-67 index was 30\% (Figure 4). The patient did not receive MDT guidance, the tumor was not treated as SS during the operation, and adjuvant treatment was not administered in time after the operation. As a result, the primary tumor recurred 7 months after the operation, and surgery was performed again. Adjuvant chemotherapy was administered after the second operation.

The estimated 5-year OS rates of the MDT-managed and non-MDT-managed groups were $31.3 \%$ and $25.0 \%$, respectively. The median OS of patients with MDT management was 46.0 months, which was longer than that of the non-MDT-managed group (18.0 months). However, when MDT management was included in the univariate analysis, there was no significant difference between the two groups (HR: 0.596, $\mathrm{P}=0.480,95 \% \mathrm{CI}$ : 0.141-2.511).

\section{Discussion}

SS has a very low incidence rate among STSs, and primary intrathoracic SS is even more rare. Since the research on primary pulmonary sarcoma by Keel et al., an increasing number of cases of intrathoracic SS have been reported, but the overall number is still low (14). In our series, pulmonary SS was the most common type (61.5\%), followed by mediastinal SS (30.8\%) and pleural SS (7.7\%). Intrathoracic SSs were more likely to develop in the lungs, similar to previous studies $(6,14,15)$. Most $(6 / 8)$ pulmonary SSs in our study were larger than $5 \mathrm{~cm}$ in diameter, and for such large tumors in the lung, oncologists should also consider the possibility of SS. The ratio of men and women was roughly equal $(1.2: 1)$ in our study, which is consistent with some previous studies $(9,16)$. We found that the average patient age was 46 years (range, 16-69 years). Previous studies have 


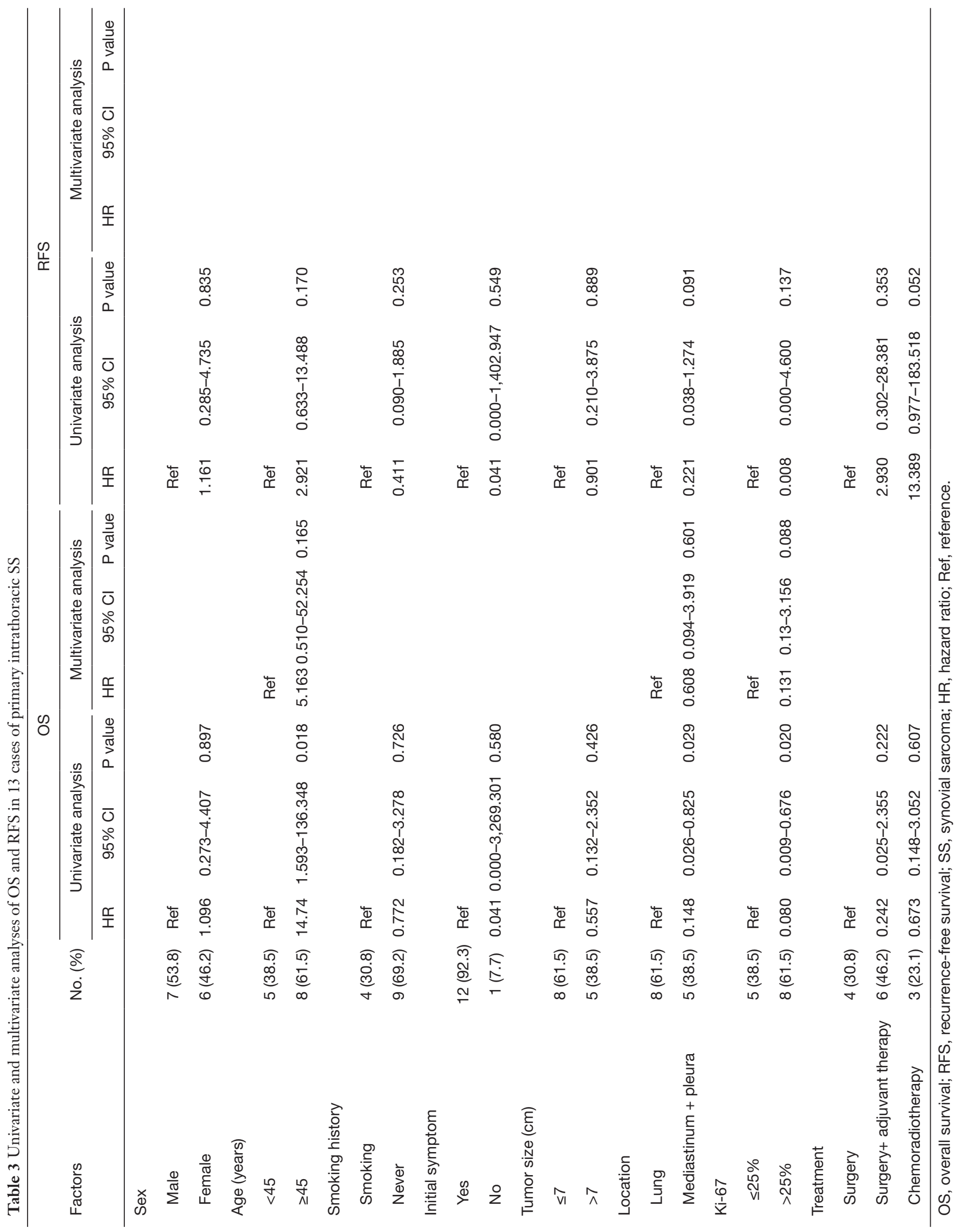



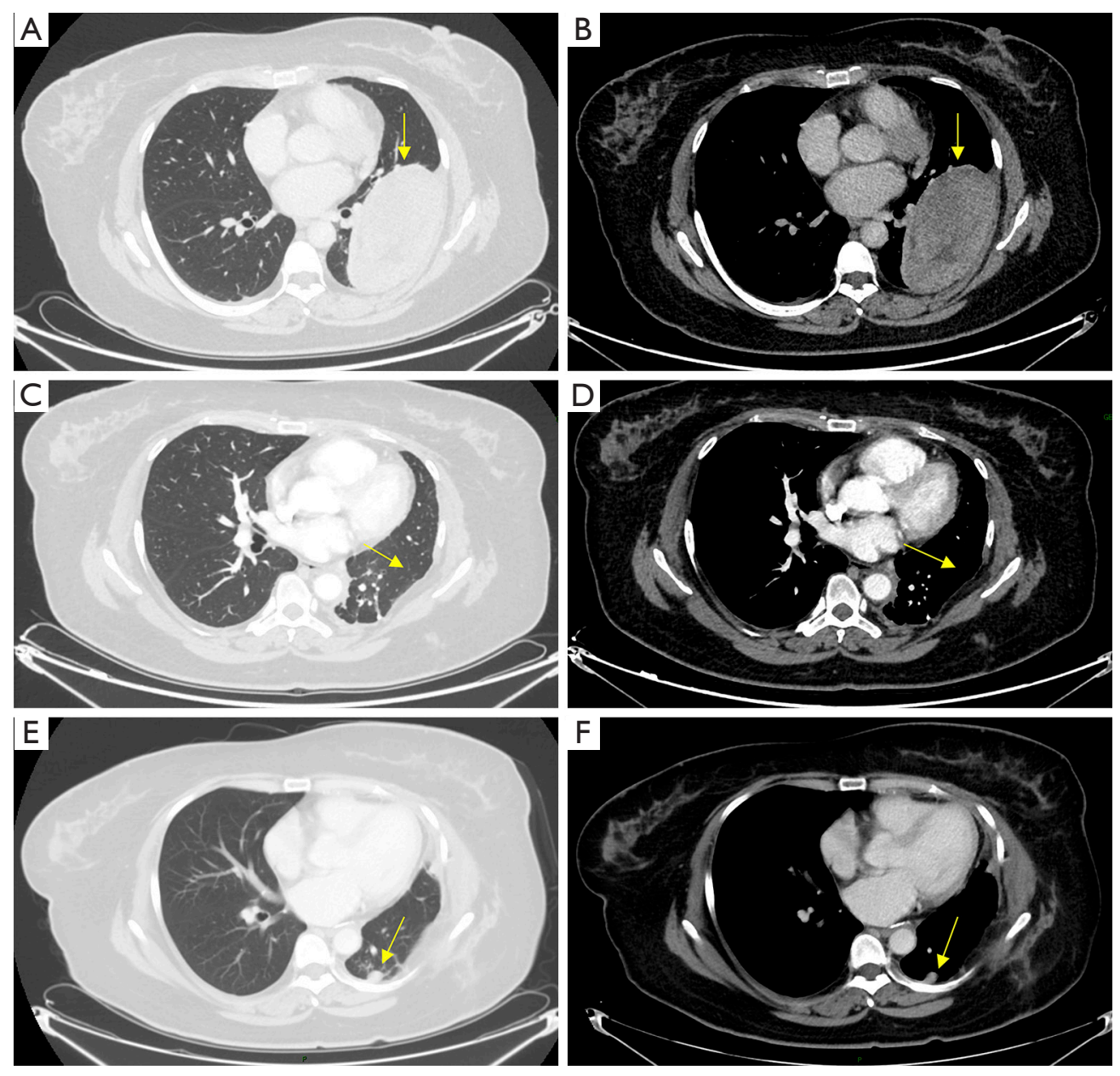

Figure 3 Chest computed tomography images of the primary pleural SS patient. (A) Non-enhanced CT (lung window): the left side of the thoracic cavity has a large tumor (arrow), which is $12.4 \mathrm{~cm} \times 6.8 \mathrm{~cm} \times 3.0 \mathrm{~cm}$ in size, with smooth edges, an unclear boundary with the pleura, incomplete compression and invasion into the adjacent lung tissue, and slight erosive damage was found in the adjacent ribs. A small amount of pleural effusion was observed. (B) Non-enhanced CT (mediastinal window; the arrow indicates the tumor). (C) Enhanced CT (lung window): postoperative changes (the arrow indicates no residual tumor). (D) Enhanced CT (mediastinal window; the arrow indicates no residual tumor). (E) Non-enhanced CT (lung window): 8 months after the previous operation, a new oval nodule (arrow) appeared behind the left costophrenic angle, with a size of $1.7 \mathrm{~cm} \times 1.0 \mathrm{~cm} \times 1.0 \mathrm{~cm}$, and the boundary was clear. (F) Non-enhanced CT (mediastinal window; the arrow indicates the tumor).

found that the average patient age is 36.5 to 47 years, which is basically consistent with our results $(6,17)$. In our series, patients over 45 years old were more likely to die from intrathoracic SS than those under 45 years old $(\mathrm{P}=0.018$, $<0.05)$. Clinically, the symptoms of primary intrathoracic SS are mostly atypical. In our series of studies, $38.5 \%$ of patients had cough and expectoration, and $38.5 \%$ of the patients had chest pain and/or dyspnea. Only one case was found incidentally during physical examination. This is similar to previous case reports and series studies $(6,7,17,18)$.
The expression of Bcl-2, CD99, TLE1, AE1/AE3, EMA, CK7, CD34, SMA and S-100 based on IHC staining can be used to assist the diagnosis $(16,19,20)$. TLE1 is a highly sensitive but not completely specific SS marker and is a screening tool for identifying patients most likely to have positive molecular genetic testing. TLE1 can also be used to identify possible keratin-negative SS for additional molecular genetic testing $(15,20)$. In addition, the Ki-67 index was higher than $10 \%$, and $61.5 \%$ of patients had a Ki-67 index higher than $25 \%$ in our study, which was 

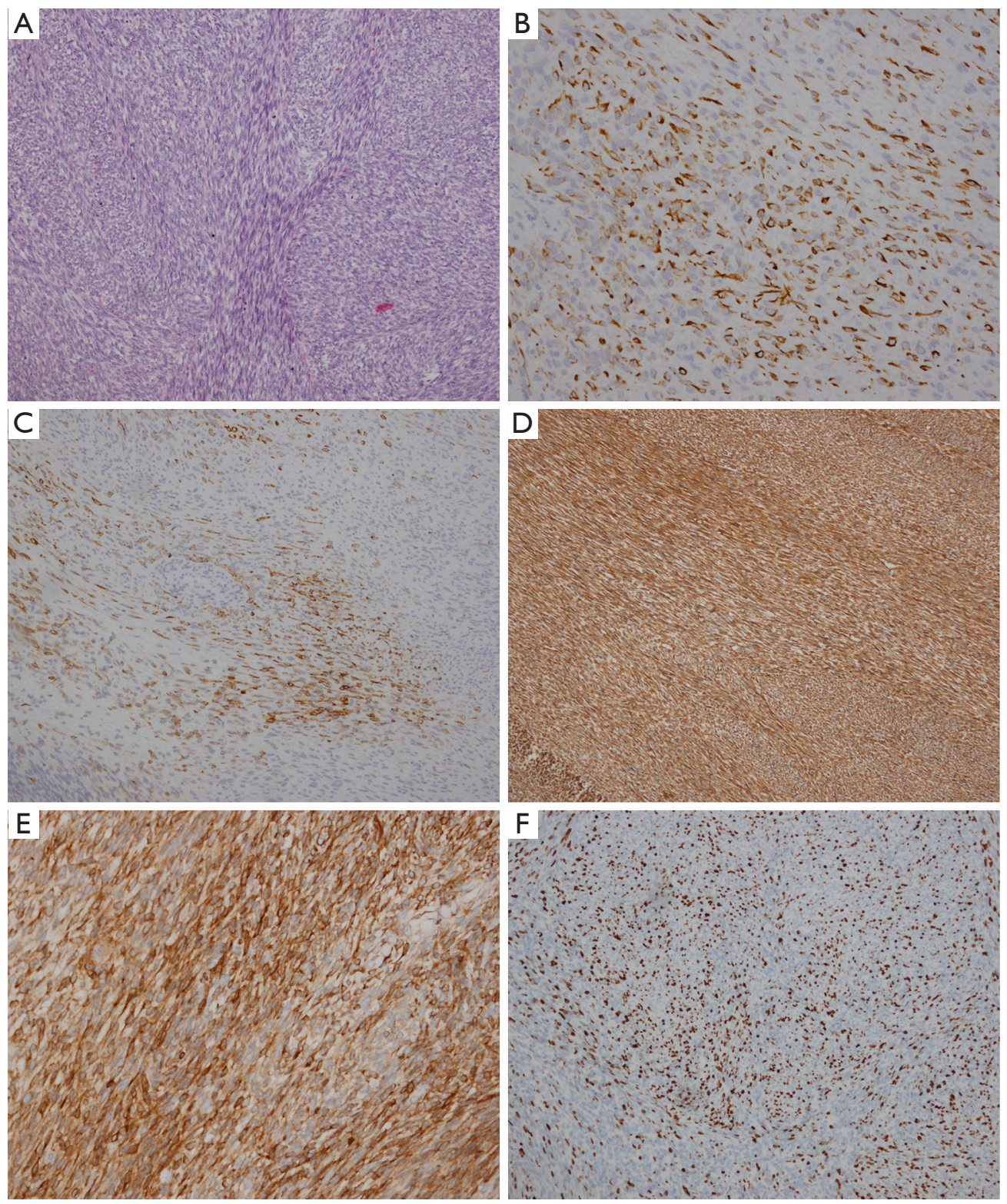

Figure 4 Microscopic characteristics. (A) Monophasic SS, the tumor is composed of well-distributed hyperchromatic fusiform cells, which are arranged in a remarkable fascicular arrangement $(\mathrm{H} \& \mathrm{E}, \times 100)$; (B) AE1/AE3 positivity (H\&E, $\times 200)$; (C) EMA positivity (H\&E, $\times 100)$; (D) Vimentin positivity (H\&E, ×100); (E) strong Bcl-2 positivity (H\&E, ×200); (F) Ki-67 30\% (H\&E, ×100). SS, synovial sarcoma.

slightly higher than that in some previous studies (21). More than $90 \%$ of SSs had chromosome translocation $\mathrm{t}(\mathrm{X} ; 18)(\mathrm{p} 11.2 ; \mathrm{q} 11.2)$, which is considered a specific marker for all morphological subtypes of primary intrathoracic SS (22). Our cases closely reflect the morphological characteristics, IHC traits and fusion gene characteristics of primary intrathoracic SS, particularly the monophasic morphology and fusion gene characteristics, which are the most characteristic differentiation points and are also the main diagnostic basis for patients if pathological results can be obtained through surgery or puncture biopsy.

In the absence of typical clinical manifestations and signs, the early diagnosis of the disease often depends on the results of imaging examinations. CT scans of mediastinal SS showed a soft tissue density shadow, lobulated appearance, clear edge, homogeneous enhancement, and liquefaction 
necrosis in the tumor, but these findings are often difficult to differentiate from those of solitary fibroma, leiomyosarcoma and rhabdomyosarcoma (23-25). In our study, 2 cases of mediastinal SS were not biopsied before surgery, one of which was submitted to an MDT meeting for discussion before surgery, and SS was considered. The preoperative diagnosis made by an MDT can greatly help surgeons determine the scope and precautions associated with tumor resection. Furthermore, the other case was only judged by the surgeon according to the results of the preoperative CT scan, which makes the judgment biased and the surgical extent insufficient. In the 3 cases of pulmonary SS, because of the appearance of a large round or irregular lobulated mass on CT scan, heterogeneous enhancement during enhanced scanning was initially highly suspected of being sarcomatoid carcinoma. When two of these cases were discussed in the MDT meeting, one was considered a pulmonary SS before the operation and finally confirmed by postoperative pathology. The diagnosis of the other depended on postoperative pathology. Most primary intrathoracic SSs feature local invasion and hematogenous metastasis but rarely lymph node metastasis $(6,16)$. Based on the hilar lymphadenopathy indicated by CT, two patients were clinically diagnosed with lung cancer by their surgeons, even though their imaging manifestations were not typical. However, the final pathological diagnosis was pulmonary SS; thus, surgeons need to pay more attention to preoperative considerations. Although rare, lymph node metastasis may occur in primary intrathoracic SS, especially pulmonary SS, which seems to prefer metastasis to the lymph nodes over other sites. The tumors of 2 patients were round or irregular, with smooth margins, clear boundaries, and notch-like changes. Both were submitted to the MDT meeting for discussion. The MDT considered that these tumors were SS before the operation and pathologically confirmed the diagnosis after the operation. Previous studies have reported that calcifications may occur in a few tumors $(11,13)$. In our study, some patients were also found to exhibit calcifications in their tumors.

In our study, $76.9 \%$ of the patients underwent surgery, and $60.0 \%$ of them received adjuvant radiotherapy, chemotherapy, targeted therapy or immunotherapy. Only $23.1 \%$ of the patients received radiotherapy and chemotherapy. Extensive radical resection was recommended for resectable lesions to protect the function of important structures $(26,27)$. However, because of their special location, most primary intrathoracic SSs had large volumes and even invaded adjacent tissues and organs, increasing the importance of multidisciplinary cooperative treatment. In the MDT meeting, numerous doctors in different professional fields made standardized and reasonable treatment plans according to the patients' pathological results and clinical characteristics and the wishes of the patients or their families. In our study, the MDT discussed 7 patients before and/or after surgery and one patient who did not receive surgery. After the MDT discussion, all patients received concurrent or sequential chemoradiotherapy, except for one patient due to physical reasons. Preoperative or postoperative radiotherapy can effectively reduce the local recurrence rate, especially for patients with large tumor volumes and a wide range of tumor beds with positive surgical margins. Palmerini et al. found that patients receiving radiotherapy had a higher 5 -year local control rate $(85 \%$ vs. $67 \%)$ than patients undergoing only surgery (28). Postoperative adjuvant chemotherapy can improve patient prognosis. A recent retrospective study of 544 patients with SS showed that adjuvant chemotherapy could prolong OS in patients with stage III SS but had no significant effect on patients with early-stage disease (29). It is difficult to widely remove tumors that are adjacent to important neurovascular structures or those invading the chest wall. It is suggested that high-dose doxorubicin drugs (e.g., a regimen of Adriamycin combined with ifosfamide) should be given for more than three cycles to high-risk patients (30). For patients who cannot undergo surgery, after MDT discussion, cytotoxic drug chemotherapy is recommended. The first-line chemotherapy scheme includes single-drug chemotherapy with anthracycline or combination therapy with ifosfamide. The combined scheme has a better effect, with a response rate of $25-60 \%$ (31). In recent years, molecular targeted therapy and immunotherapy have shown great advantages in the fight against tumors, which is also reflected in the treatment of SS. A phase III randomized clinical trial $(n=38)$ showed that pazopanib can improve the prognosis of SS patients and prolong the median progression-free survival duration by 3 months (4.1 vs. 1.0 months) (32). The overall response rate of 21 patients with advanced STS treated with palliative apatinib therapy was $71.4 \%$, and the average effective response interval of 6 patients with SS was 5.8 months, indicating that apatinib is expected to improve the survival of patients with advanced SS (33). Some scholars have also implemented immunotherapy for STSs, including SSs. Eleven patients (29.7\%) showed different degrees of tumor reduction, 6 of whom achieved PR, and the 12-week progression-free 
survival rate was $44 \%$ (34). In our study, 2 patients were recommended for immunotherapy by the MDT when they were resistant to chemotherapy and progressed. One patient has already survived for 39 months.

The 5 -year OS rate of our series is close to that of a previous study (30\% vs. 30.0-31.6\%) (6,35) and lower than that of systemic SS (90\% in children and $50-60 \%$ in adults) (36). This may be related to the tumor directly affecting or invading the important anatomical structures of the heart and lung and to the large tumor volume at diagnosis. A total of $69 \%$ of patients with mediastinal SS die within 3 years after diagnosis (37). Due to the limited number of cases included in our study, MDT treatment did not show an independent effect on the prognosis of patients. However, the median OS of patients who were managed by an MDT was longer than that of patients who were not managed by an MDT (46.0 vs. 18.0 months). This is mainly due to the relatively accurate diagnosis, standardized surgical resection and reasonable therapeutic schedule established by the MDT. Similarly, some previous studies also found that lung cancer patients discussed at MDT meetings had significantly longer survival times than those not discussed (38-40).

Our research is subject to limitations. First, because of its retrospective, single-center nature and other characteristics, there were inevitable biases in patient selection and statistical analysis. Second, because of the low incidence rate of SS in the thoracic cavity, the number of patients was very limited, leading to bias in the statistical analysis and preventing convincing conclusions. For rare tumors, such as primary intrathoracic SS, larger sample sizes are needed to further summarize their characteristics and provide guidance for diagnosis and treatment. Collecting such samples will be one of the directions of our future work.

\section{Conclusions}

Primary intrathoracic SS has both common and unique pathogenic, clinical histopathological and immunohistochemical features. Although the incidence rate of primary intrathoracic SS is low, it is difficult to diagnose and can be easily misdiagnosed. FISH detection and RT-PCR are powerful tools for diagnosis when supplemented by immunohistochemistry. Monophasic morphology and fusion gene characteristics are the main basis of diagnosis of primary intrathoracic SS. When treating primary intrathoracic SS, it is first recommended to remove the tumor as thoroughly as possible. Preoperative or postoperative adjuvant chemotherapy and radiotherapy, especially adriamycin combined with an ifosfamide regimen, can effectively reduce local recurrence and improve prognosis. Molecular targeted therapy and immunotherapy have great prospects in the treatment of SS. MDTs can play an important role in the diagnosis and treatment of patients, and it is worth recommending in the management of primary intrathoracic SS.

\section{Acknowledgments}

We thank all the staff in the Department of Thoracic Surgery for their support during the study.

Funding: This study was supported by the Institutional Fundamental Research funds (2018PT32033) and the Ministry of Education Innovation Team Development Project (IRT-17R10).

\section{Footnote}

Reporting Checklist: The authors have completed the STROBE reporting checklist. Available at http://dx.doi. org/10.21037/jtd-20-2887

Data Sharing Statement: Available at http://dx.doi. org/10.21037/jtd-20-2887

Conflicts of Interest: All authors have completed the ICMJE uniform disclosure form (available at http://dx.doi. org/10.21037/jtd-20-2887). The authors have no conflicts of interest to declare.

Etbical Statement: The authors are accountable for all aspects of the work in ensuring that questions related to the accuracy or integrity of any part of the work are appropriately investigated and resolved. This study was approved by the Ethics Committee of National Cancer Center/Cancer Hospital, Chinese Academy of Medical Sciences and Peking Union Medical College (approval number: 20/210-2406). The requirement for informed consent was waived because of the retrospective nature of this study.

Open Access Statement: This is an Open Access article distributed in accordance with the Creative Commons Attribution-NonCommercial-NoDerivs 4.0 International License (CC BY-NC-ND 4.0), which permits the noncommercial replication and distribution of the article with 
the strict proviso that no changes or edits are made and the original work is properly cited (including links to both the formal publication through the relevant DOI and the license). See: https://creativecommons.org/licenses/by-nc-nd/4.0/.

\section{References}

1. Jemal A, Siegel R, Xu J, et al. Cancer statistics, 2010. CA Cancer J Clin 2010;60:277-300.

2. Pan $M$, Merchant $M$. Risk factors including age, stage and anatomic location that impact the outcomes of patients with synovial sarcoma. Med Sci (Basel) 2018;6:21.

3. Cadman NL, Soule EH, Kelly PJ. Synovial sarcoma; An analysis of 134 tumors. Cancer 1965;18:613-27.

4. Song S, Park J, Kim HJ, et al. Effects of Adjuvant Radiotherapy in Patients With Synovial Sarcoma. Am J Clin Oncol 2017;40:306-11.

5. Krugman ME, Rosin HD, Toker C. Synovial sarcoma of the head and neck. Arch Otolaryngol 1973;98:53-4.

6. Lan T, Chen H, Xiong B, et al. Primary pleuropulmonary and mediastinal synovial sarcoma: a clinicopathologic and molecular study of 26 genetically confirmed cases in the largest institution of southwest China. Diagn Pathol 2016;11:62.

7. Falkenstern-Ge RF, Kimmich M, Grabner A, et al. Primary pulmonary synovial sarcoma: a rare primary pulmonary tumor. Lung 2014;192:211-4.

8. García JS, Ramos MB, Fontan EM, et al. Primary pulmonary synovial sarcoma: a rare neoplasm. Kardiochir Torakochirurgia Pol 2016;13:148-9.

9. Essary LR, Vargas SO, Fletcher CD. Primary pleuropulmonary synovial sarcoma: reappraisal of a recently described anatomic subset. Cancer 2002;94:459-69.

10. Yamaki $M$, Yonehara S, Noriyuki T. Large primary pleural synovial sarcoma with severe dyspnea: a case report. Surg Case Rep 2017;3:29.

11. de Silva MV, McMahon AD, Paterson L, et al. Identification of poorly differentiated synovial sarcoma: a comparison of clinicopathological and cytogenetic features with those of typical synovial sarcoma. Histopathology 2003;43:220-30.

12. Klebe S, Prabhakaran S, Hocking A, et al. Pleural malignant mesothelioma versus pleuropulmonary synovial sarcoma: a clinicopathological study of 22 cases with molecular analysis and survival data. Pathology 2018;50:629-34.

13. Ishida H, Fujino T, Taguchi R, et al. Primary pulmonary synovial sarcoma with calcification: A case report. Thorac
Cancer 2019;10:2040-4.

14. Keel SB, Bacha E, Mark EJ, et al. Primary pulmonary sarcoma: a clinicopathologic study of 26 cases. Mod Pathol 1999;12:1124-31.

15. Lino-Silva LS, Flores-Gutierrez JP, Vilches-Cisneros $\mathrm{N}$, et al. TLE1 is expressed in the majority of primary pleuropulmonary synovial sarcomas. Virchows Arch 2011;459:615-21.

16. Zeren H, Moran CA, Suster S, et al. Primary pulmonary sarcomas with features of monophasic synovial sarcoma: a clinicopathological, immunohistochemical, and ultrastructural study of 25 cases. Hum Pathol 1995;26:474-80.

17. Godil A, Ali Mallick MS, Khetpal A, et al. Primary Synovial Sarcoma of the Mediastinum in an 18-year old male: A case report. J Pak Med Assoc 2018;68:1708-10.

18. Bhattacharya D, Datta S, Das A, et al. Primary pulmonary synovial sarcoma: A case report and review of literature. Int J Appl Basic Med Res 2016;6:63-5.

19. Salah S, Salem A. Primary synovial sarcomas of the mediastinum: a systematic review and pooled analysis of the published literature. ISRN Oncol 2014;2014:412527.

20. Knösel T, Heretsch S, Altendorf-Hofmann A, et al. TLE1 is a robust diagnostic biomarker for synovial sarcomas and correlates with $\mathrm{t}(\mathrm{X} ; 18)$ : Analysis of 319 cases. Eur J Cancer 2010;46:1170-6.

21. Okamoto S, Hisaoka M, Daa T, et al. Primary pulmonary synovial sarcoma: a clinicopathologic, immunohistochemical, and molecular study of 11 cases. Hum Pathol 2004;35:850-6.

22. Park JS, Min BR, Park SH, et al. Primary pulmonary biphasic synovial sarcoma confirmed by molecular detection of a SYT-SSX2 fusion gene: report of 1 case. Korean J Intern Med 2010;25:331-6.

23. Loscertales J, Trivino A, Gallardo G, et al. Primary monophasic synovial sarcoma of the pleura: diagnosis and treatment. Interact Cardiovasc Thorac Surg 2011;12:885-7.

24. Suster S, Moran CA. Primary synovial sarcomas of the mediastinum: a clinicopathologic, immunohistochemical, and ultrastructural study of 15 cases. Am J Surg Pathol 2005;29:569-78.

25. Chirmade PC, Parikh S, Anand A, et al. Primary pleuropulmonary synovial sarcoma with brain metastases in a paediatric patient: an unusual presentation. Adv Respir Med 2017;85:206-10.

26. Nishiyama N, Iwata T, Izumi N, et al. Aggressive repeat 
surgery for a recurrent synovial sarcoma in the pleura. Ann Thorac Cardiovasc Surg 2009; 15:46-9.

27. Yaser S, Salah S, Al-Shatti M, et al. Prognostic factors that govern localized synovial sarcoma: a single institution retrospective study on 51 patients. Med Oncol 2014;31:958.

28. Palmerini E, Staals EL, Alberghini M, et al. Synovial sarcoma: retrospective analysis of 250 patients treated at a single institution. Cancer 2009;115:2988-98.

29. Vining CC, Sinnamon AJ, Ecker BL, et al. Adjuvant chemotherapy in resectable synovial sarcoma. J Surg Oncol 2017;116:550-8.

30. Stacchiotti S, Van Tine BA. Synovial Sarcoma: Current Concepts and Future Perspectives. J Clin Oncol 2018;36:180-7.

31. Noujaim J, Constantinidou A, Messiou C, et al. Successful Ifosfamide Rechallenge in Soft-Tissue Sarcoma. Am J Clin Oncol 2018;41:147-51.

32. van der Graaf W'T, Blay JY, Chawla SP, et al. Pazopanib for metastatic soft-tissue sarcoma (PALETTE): a randomised, double-blind, placebo-controlled phase 3 trial. Lancet 2012;379:1879-86.

33. Zhu B, Li J, Xie Q, et al. Efficacy and safety of apatinib monotherapy in advanced bone and soft tissue sarcoma: An observational study. Cancer Biol Ther 2018;19:198-204.

34. Tawbi HA, Burgess M, Bolejack V, et al. Pembrolizumab in advanced soft-tissue sarcoma and bone sarcoma

Cite this article as: $\mathrm{He} \mathrm{H}$, Yang L, Peng Y, Liu L, Liu L, Xue Q, Gao S. The value of multidisciplinary team (MDT) management in the diagnosis and treatment of primary intrathoracic synovial sarcomas: a single-center experience. J Thorac Dis 2021;13(2):600-612. doi: 10.21037/jtd-20-2887
(SARC028): a multicentre, two-cohort, single-arm, openlabel, phase 2 trial. Lancet Oncol 2017;18:1493-501.

35. Bégueret H, Galateau-Salle F, Guillou L, et al. Primary intrathoracic synovial sarcoma: a clinicopathologic study of $40 \mathrm{t}(\mathrm{X} ; 18)$-positive cases from the French Sarcoma Group and the Mesopath Group. Am J Surg Pathol 2005;29:339-46.

36. Hartel PH, Fanburg-Smith JC, Frazier AA, et al. Primary pulmonary and mediastinal synovial sarcoma: a clinicopathologic study of 60 cases and comparison with five prior series. Mod Pathol 2007;20:760-9.

37. Terra SB, Aesif SW, Maleszewski JJ, et al. Mediastinal synovial sarcoma: clinicopathologic analysis of 21 cases with molecular confirmation. Am J Surg Pathol 2018;42:761-6.

38. Bydder S, Nowak A, Marion K, et al. The impact of case discussion at a multidisciplinary team meeting on the treatment and survival of patients with inoperable nonsmall cell lung cancer. Intern Med J 2009;39:838-41.

39. Forrest LM, McMillan DC, McArdle CS, et al. An evaluation of the impact of a multidisciplinary team, in a single centre, on treatment and survival in patients with inoperable non-small-cell lung cancer. Br J Cancer 2005;93:977-8.

40. Bilfinger TV, Albano D, Perwaiz M, et al. Survival Outcomes Among Lung Cancer Patients Treated Using a Multidisciplinary Team Approach. Clin Lung Cancer 2018;19:346-51. 